\title{
Design on Digital Resources Reading Tendency Investigate System of College Students
}

\author{
Li Ma ${ }^{1, a}$, YuYang $\mathrm{Zu}^{2, \mathrm{~b}}$ \\ ${ }^{1}$ School of Education and Sports, Bohai University, Jinzhou, 121013, China \\ ${ }^{2}$ College of Foreign Languages, Bohai University, Jinzhou, 121013, China \\ a88228471@qq.com, b1143119122@qq.com
}

Keywords: college students; digital resources; reading tendency; Investigate system

\begin{abstract}
Reading is the process of college students' understanding of the world and their own knowledge, is the main channel to expand the knowledge perspective, and is the main means to enhance the comprehensive cultural quality. In the electronic information era, the investigation of digital resources in college students' reading tendency is the starting point to solve the problem of College Students' reading accuracy and efficiency, but also to guide the college students' reading and reading ability premise design strategy. This paper is based on the Struts2 framework, which includes three aspects: input verification design, functional framework design and data persistence design. This design gives full play to the advantages of Struts2 framework is clear and strong, timely treatment of abnormal interceptor and modular application etc..
\end{abstract}

\section{Introduction}

Reading is one of the unique spiritual and cultural activities of human beings. The history of reading is also the history of human civilization and the development of human spirit. At present, the national reading has become one of the important measures to improve the national quality and promote social harmony and progress. With the development of information technology, profound changes have taken place in the way of reading and behavior. Digital reading has become an important way of reading [1]. Digital reading is to read the digital, mainly has two meanings: one is the object of reading, digital, is read in a digital way, such as books, online novels, electronic map, digital photos, blogs and web pages; The two is, reading digital, is the carrier and the reading is not flat paper, but with the screen display of electronic devices, such as PC, PDA, MP3, MP4, notebook computers, mobile phones and readers, etc.. Compared with traditional paper publications, digital electronic publications have many advantages, such as large storage, convenient retrieval, easy to save and low cost.

Struts2 is the next generation of Struts products. Is based on struts and WebWork technology on the merger, the formation of a new MVC framework. The new Struts2 architecture and Struts1 architecture vary greatly. Struts2 to WebWork as the core, the interceptor mechanism to deal with the user's request, the business logic controller can complete off the Servlet and API, so Struts2 can be understood as the update product WebWork. Because Struts2 and Struts1 have a lot of change, but with respect to WebWork, Struts2 is only a small change. Struts2 goal is to hope that the original use of Struts1, WebWork developers, can be a smooth transition to the Struts2 framework. The advantages of Struts2 are mainly embodied in [2]: Based on the MVC architecture framework, clear structure, clear development process; strong interceptor, Struts2 interceptor is a Action level of AOP, many properties are realized by the interceptor; easier to test, Struts2 Action is a simple POJO, you can easily write test cases.

Reading is the process of understanding the world, understanding the society and understanding oneself, it is the main channel to expand the visual field of knowledge, and it is also the main means to enhance the comprehensive cultural quality. It is the key to study and solve the problems of College Students' correct reading and efficient reading to clarify the problems of "why to read", "what should be read" and "how to read". In the electronic information era, carries on the investigation to the digital resources of College Students' reading tendency is the starting point to 
solve the analysis accuracy and efficiency problems of college students in reading, but also guide the college students' reading and reading ability to the fundamental premise of design strategy [3]. In this paper, based on the Struts2 framework for system design, digital information resources for college students to read the survey provides information solutions.

\section{Input Validation Design}

Struts2 input check is divided into client check and server check. The client is in check through the JavaScript data collection preliminary filter page, JavaScript is a very powerful web page script, regular expressions. The main function of the client check is to prevent the normal browser of the wrong input, the input can only be a preliminary filter for the user's malicious behavior, the client is unable to verify. Therefore, the client can not be replaced by the end of the server checksum checksum server is used in the server side of the program code or configuration file for the user input data validation. Server side check is the last line of defense to prevent illegal data throughout the application, mainly through the application programming [4,5].

Input validation is a kind of presentation layer data processing, so it should be provided by the MVC framework. The Struts2 framework provides a very powerful input validation system, through the input validator built-in Struts2, without writing any input validation code, can complete the most check, and can complete the client and server check check. If the input validation rules used are particularly complex, Struts2 also allows you to perform a custom check by overriding the validate method. In addition, the Struts2's openness allows the developer to provide a custom validator. Struts2 input verification process shown in Fig. 1.

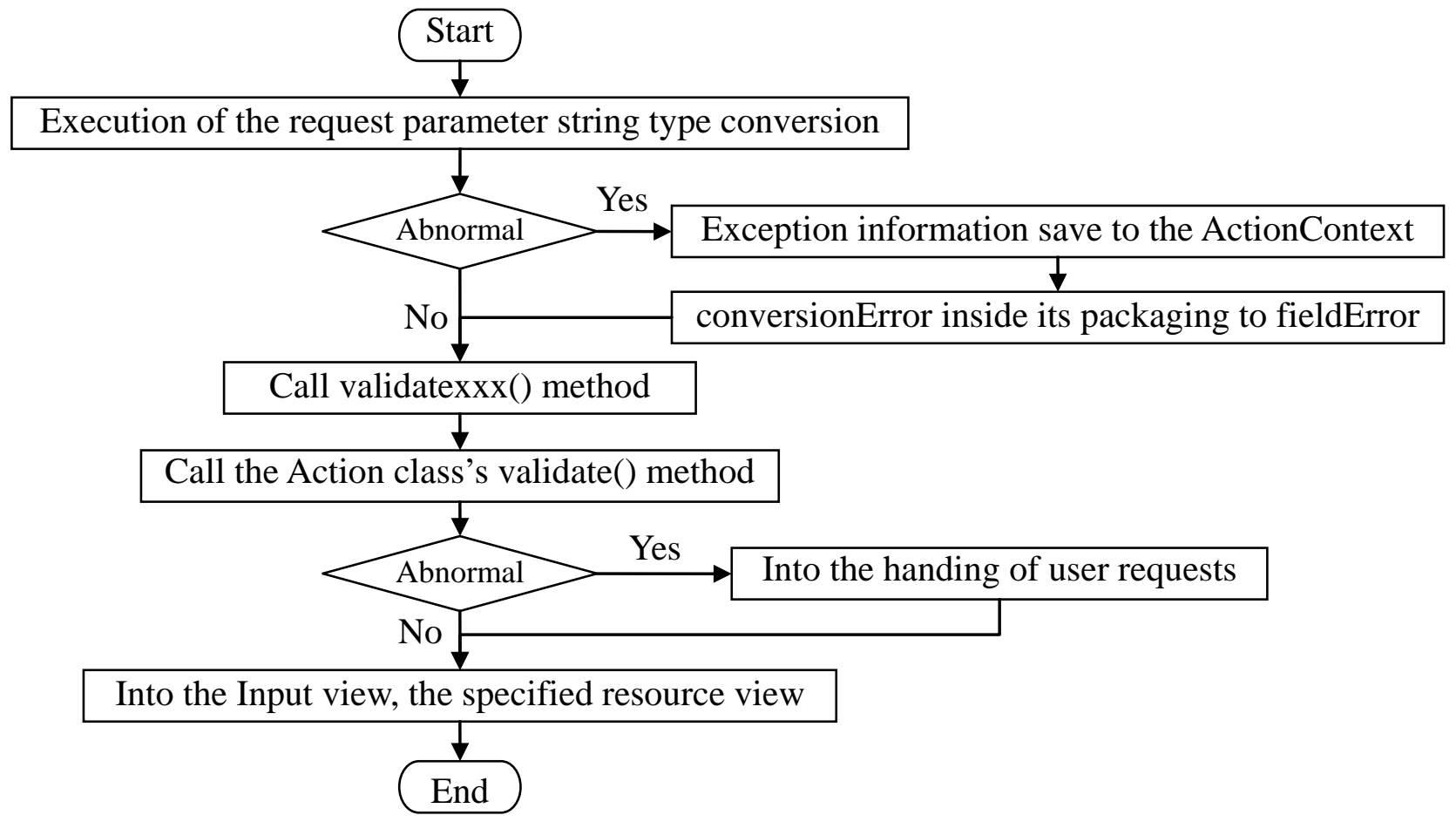

Fig .1. Input inspection process of Struts2

According to Fig. 1, the input validation of Struts2 is usually done in the following steps: a type converter performs a type conversion on the string's request parameters and sets the values to the Action property value; Type conversion process if abnormal, abnormal information will be saved to the ActionContext, the conversionError interceptor package it into the fieldError, the next step. If not abnormal, the direct execution of the next step; by calling the validateXxx() method, the reflection method is $\mathrm{Xxx}$ which is the processing logic of user request corresponding to the name of the Action class; call validate () method; if abnormal, directly into the input view of the specified view resources (for verification method. The input must be specified in the configuration file). If no 
exception occurs, the processing method of the user request is transferred, and then transferred to the view resource specified in the input view.

\section{Functional Framework Design}

The common method of functional design is simulation. The modular design idea is a kind of important, a complicated system is decomposed into smaller, simpler and easier to create and modify the part, on the one hand, each module is relatively independent and can be separately designed, on the other hand, to illustrate the relationships between the modules through a certain the way. Under the constraints of these relations, each module forms a unified whole, complete the functions of the system.

The function frame structure is the result of the simulation, it is the function of the system decomposition, according to the function of the relationship between the graph, the graph of each rectangular box is called a function module. Each subsystem of the management information system can be regarded as the function of the lower level of the system target. Function decomposition process from large to small, from coarse to fine, from top to bottom. Conceptually speaking, the upper layer functions to control the lower layer function, the more the upper layer function is more general, the more the lower layer function is more concrete. The process of functional decomposition is a process from abstract to concrete, from complex to simple. The function module according to the specific circumstances of larger or smaller, the decomposition of function modules can be minimum each process in a program, and the larger function module can be a set of program to complete a task. The results of the functional framework design of College Students' Reading Tendency of digital resources are shown in Fig. 2.

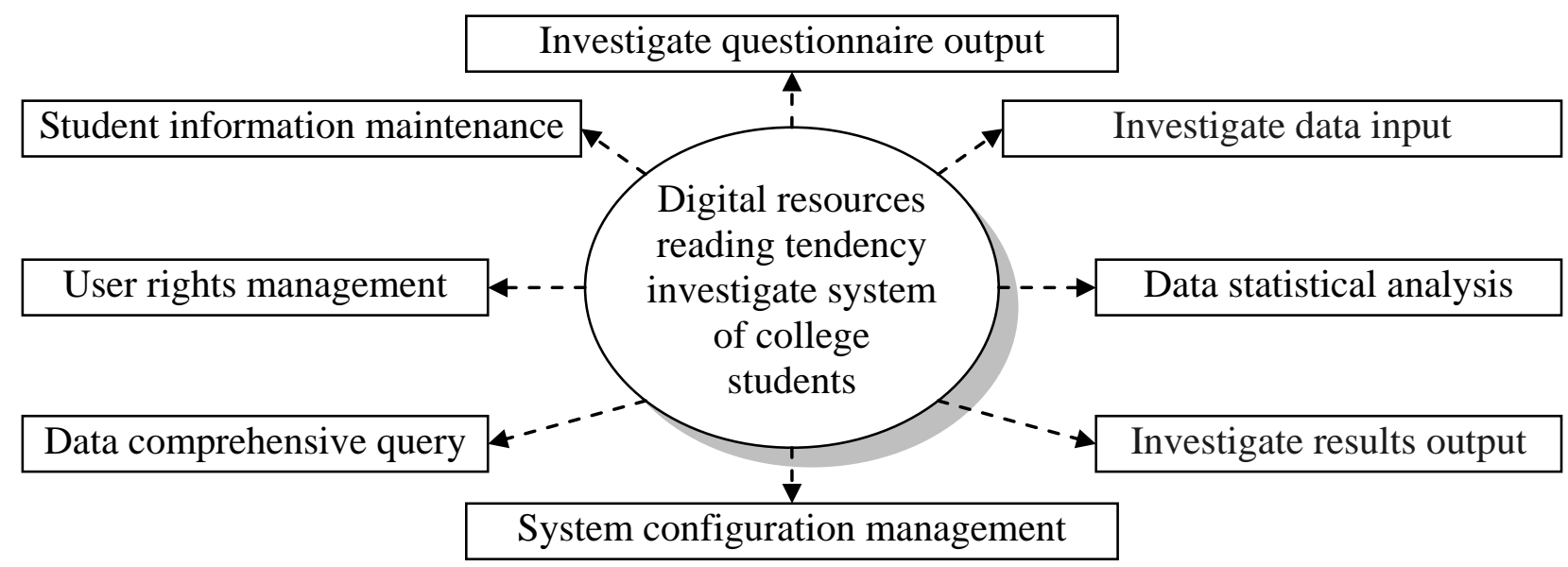

Fig. 2. Functional framework on digital resources reading tendency investigate system of college students

The functional architecture shown in Fig. 2 includes a total of 8 functions. Among them, Student information maintenance, for students of the personal information management, including input, modify and delete; Investigate questionnaire output, will form the printer output, or output to a file using the PDF Investigate data input print survey; and the input data of the questionnaire to the computer system, modify and delete functions Data statistical analysis, is also provided; related using the method of mathematical statistics, statistical analysis of survey data; Investigate results output, the statistical analysis results of output, output, including screen printer or file; Data comprehensive query, enter the query, get the query result; System configuration management, for the management of software operation the User rights management environment variable, Assign roles to users and grant different permissions to users of different roles.

\section{Data Persistence Design}

Java is the most commonly used Persistence technology in the field of Hibernate. Hibernate is an open source object relational mapping framework, which is a lightweight object encapsulation for 
JDBC, so that Java programmers can use the object programming thinking to manipulate the database. Not only provides a mapping from Java to the data table, but also provides a data query and recovery mechanism [6]. Compared to using JDBC and SQL to manually manipulate the database, Hibernate reduces the workload of operating the database. In the process of system design, each database table needs to be persisted, because the space is larger, this paper takes the "student entity" as an example to illustrate the design process. Based on the SQL Server database management system, the logical structure of the "student entity" is shown in Table 1.

Table 1. Logical structure design on college students basic information table

\begin{tabular}{clcc|clcc}
\hline No & Fields Name & Type & Bytes & No & Fields Name & Type & Bytes \\
\hline 1 & StudentNumber & Varchar & 10 & 12 & SpecialtyCode & Varchar & 6 \\
2 & StudentName & Varchar & 20 & 3 & SpecialtyName & Varchar & 50 \\
3 & BirthDate & Datetime & 4 & 14 & MobilePhone & Varchar & 50 \\
4 & StudentGender & Char & 2 & 15 & E-mail & Varchar & 50 \\
5 & SchoolYear & Char & 4 & 16 & ReadingTools & Varchar & 50 \\
6 & CollegeCode & Varchar & 6 & 17 & ReadingPatterns & Varchar & 100 \\
7 & CollegeName & Varchar & 50 & 18 & PersonalProfile & Varchar & 500 \\
\hline
\end{tabular}

(1) Persistence class design. Persistence class is used to complete the access to the database, the data will be saved to the database or read data from the database. An instance of a persistent class is in one of three states, Transient, Persistent and Detached, which is related to the persistence context. The save() method converts the temporary object to a persistent object; the update() method converts the detached object to a persistent object; the close() method turns the persistent object into a detached object; the delete() As a temporary object or persistent object into a temporary object;load() method will be temporary object into a detached object. A persistent object, commonly known as a PO (Persistent Object), is a simple object used to load data, wrapping functions, processing, values, database access, and any other object that can be created with Java code. JavaBean without having to worry about changes. PO is defined by an entity attribute, an associated entity definition, and a getter / setter method. Student entity persistence class design code is as follows:

\begin{tabular}{l|l}
\hline public class studentsInformation implements & private String personalProfile; \\
Serializable & private String mobilePhone; \\
\{ // Entity attribute list & private String e-mail; \\
private String studentNumber; & private String readingTools; \\
private String studentName; & private String collegeCode; \\
private Date birthDate; & private String collegeName; \\
private String studentGenderr; & // Set methods of getter and setter \\
private String schoolYear; & // getter and setter methods of entity attribute \\
private String collegeCode; & public string setStudentNumber (string studentNumber) \\
private String collegeName; & $\{$ this.studentNumber =studentNumber; $\}$ \\
private String specialtyCode; & public studentsInformation getStudentNumber() \\
private String specialtyName; & $\{$ return this.studentNumber; $\}$ \\
private String readingPatterns; & // Getter and setter methods for omitting other attributes
\end{tabular}

(2) Mapping file design. Through a series of XML elements of the configuration, the persistent class mapping to the database table, and the persistence of the class attribute mapping data columns. The mapping file usually consists of four elements, <hibernate-mapping $>$ is the root element of the mapping file, contains several optional attributes; <class $>$ defines a persistent class mapping relationship with the data table; <id> mapping attribute to the database table primary key $<$ Property $>$ Map the mapping between the attributes of the persistent class and the database table fields; <composite-id> maps the union primary key of multiple attributes; <generator> defines the built-in generator, , The UUID containing the IP address and the JVM boot time, the Identity key 
generated by the underlying database, the Sequence sequence identifier provided by the underlying database, the Hilo identifier generated by the high / low algorithm, and the underlying database The generated native identifier, and so on. The mapping file for the student entity corresponding to the PO implementation is as follows:

\begin{tabular}{|c|c|}
\hline $\begin{array}{l}<\text { ?xml version="1.0" encoding="UTF-8"?> } \\
<\text { !--Hibernate Mapping file DTD information } \\
\text { (omitted) -- > } \\
<\text { !-- Hibernate Map file root element -- > } \\
<\text { hibernate-mapping package="org.yeeku.model" > } \\
<\text { !-- Each class is mapped to a persistent class -- > } \\
<\text { calss name= "indicators" table= "indicators" > } \\
\text { // "studentNumber" as the primary key field } \\
<\text { composite-id }> \\
<\text { key-property name="studentNumber"/> } \\
<\text { composite-id }> \\
<\text { !-- Mapping properties in class -- > } \\
<\text { property name="studentNumber" type="String"/> }\end{array}$ & $\begin{array}{l}\text { <property name="studentName" type="String"/> } \\
\text { <property name="birthDate" type="Date"/> } \\
\text { <property name="studentGenderr" type="String"/> } \\
\text { <property name="schoolYear" type="String"/> } \\
\text { <property name="collegeCode" type="String"/> } \\
\text { <property name="collegeName" type="String"/> } \\
\text { <property name="specialtyCode" type="String"/> } \\
\text { <property name="specialtyName" type="String"/> } \\
\text { <property name="mobilePhone" type="String"/> } \\
\text { <property name="e-mail" type="String"/> } \\
\text { <property name="readingTools" type="String"/> } \\
\text { <property name="collegeCode" type="String"/> } \\
\text { <property name="collegeName" type="String"/> }\end{array}$ \\
\hline
\end{tabular}

\section{Acknowledgement}

This work is supported by 2013 annual social science planning fund project of Liaoning province (L13CTQ012): Research on investigation and cultivation for college students' reading tendency of digital resources; 2014 annual education science planning of Liaoning province (JG14DB019): Strategy research on teachers' teaching mode transformation and teaching ability in digital environment.

\section{References}

[1] T. Y. Gao, "College Students' Reading Tendency of digital resources and the strategy of Library Service Innovation," The Library Journal of Henan, vol. 35, no. 6, pp. 50-53, 2015.

[2] X. B. Chen, "Advantages of struts2," Dhttp://xiaobing259-163-com.iteye.com/blog/1530960, 2016-1-8.

[3] Y. S. Teng, "Investigation on the Digital Reading Tendency of College Students in Nationwide Reading: An Example of Shanghai Theatre Academy," Digital Library Forum, vol. 11, no. 6, pp. 45-48, 2016.

[4] C. H. Nie, "Application Research of data input processing based on Struts2," Information Technology and Informatization, vol. 41, no. 10, pp. 51-55, 2016.

[5] X. M. Li, "Based on the Input Validation Functions Struts2 Framework Implementation and Application," Computer Knowledge and Technology, vol. 9, no. 35, pp. 8201-8202, 2013.

[6] W. J. Tang, L. N. Chen, "Performance Optimization Scheme Research based on the Hibernate Lasting Layer," Intelligent Computer and Applications, vol. 2, no. 1, pp. 56-58, 2012. 